\title{
Insights into Chronic Obstructive Pulmonary Disease as Critical Risk Factor for Cardiovascular Disease
}

This article was published in the following Dove Press journal: International Journal of Chronic Obstructive Pulmonary Disease

\author{
Pere Almagro (D) \\ Ramon Boixeda $\left.{ }^{2}\right)^{2}$ \\ Jesús Diez-Manglano $\mathbb{1 D}^{3}$ \\ María Gómez-Antúnez ${ }^{4}$ \\ Francisco López-García \\ Jesús Recio (iD ${ }^{6}$ \\ 'Multimorbidity Patient Unit, Service of \\ Internal Medicine, Hospital Universitari \\ Mútua de Terrassa, Terrassa, Barcelona, \\ Spain; ${ }^{2}$ Service of Internal Medicine, \\ Hospital de Mataró-CSDM, Barcelona, \\ Mataró, Spain; ${ }^{3}$ Service of Internal \\ Medicine, Hospital Royo Villanova, \\ Zaragoza, Spain; ${ }^{4}$ Service of Internal \\ Medicine, Hospital General Universitario \\ Gregorio Marañón, Madrid, Spain; \\ ${ }^{5}$ Service of Internal Medicine, Hospital \\ General Universitario de Elche, Alicante, \\ Elche, Spain; ${ }^{6}$ Service of Internal \\ Medicine, Hospital Quironsalud València, \\ Valencia, Spain
}

Correspondence: Pere Almagro Unit of Complex Chronic Patients, Service of Internal Medicine, Hospital Universitari Mútua de Terrassa, Plaça del Doctor Robert 5, Terrassa, Barcelona E-0822I

Email 19908pam@comb.cat

\begin{abstract}
In patients with chronic obstructive pulmonary disease (COPD), cardiovascular comorbidities are highly prevalent and associated with considerable morbidity and mortality. This coincidence is increasingly seen in the context of a "cardiopulmonary continuum" rather than being simply attributed to shared risk factors, in particular, cigarette smoking. Both disease entities are centrally linked to systemic inflammation as well as aging, arterial stiffness, and several common biomarkers that led to the development of pulmonary hypertension, left ventricular diastolic dysfunction, atherosclerosis, and reduced physical activity and exercise capacity. For these reasons, COPD should be considered an independent factor of high cardiovascular risk, and efforts should be directed to early identification of cardiovascular disease (CVD) in COPD patients. Assessment of the overall cardiovascular risk is especially important in patients with severe exacerbation episodes, and the same therapeutic target levels for glycosylated hemoglobin, low-density lipoprotein cholesterol (LDL-C), or blood pressure than those recommended by clinical practice guidelines for patients at high cardiovascular risk, should be achieved. In this review, we will discuss the most recent evidence of the role of COPD as a critical cardiovascular risk factor and try to find new insights and potential prevention strategies for this disease.
\end{abstract}

Keywords: chronic obstructive pulmonary disease, cardiovascular disease, cardiovascular risk factors, inflammation, ischemic heart disease, heart failure

\section{Introduction}

Chronic obstructive pulmonary disease (COPD) is a major public health problem with impressive statistics. The Global Burden of Disease Study reports a prevalence of 251 million cases of COPD globally in 2016, with 3.17 million deaths caused by the disease in 2015 (5\% of all deaths globally in that year). ${ }^{1,2}$ Moreover, COPD is likely to increase in the coming years due to higher smoking prevalence and aging populations in many countries. Cardiovascular diseases (CVD) are highly prevalent in patients with COPD and are a clinically relevant cause of morbidity and mortality. In a well-characterized cohort of 213 patients with moderate to very severe COPD, one or more comorbidities were present in $97.7 \%$ of the patients, and four or more in $53.5 \%{ }^{3}$ However, establishing the true prevalence of individual comorbidities in COPD and their relationship with the prognosis of the disease is confounded by different factors, including shared risk factors for COPD and several comorbidities; underdiagnosis of both COPD and comorbid conditions, and the symptoms overlap between COPD and comorbidities. ${ }^{4}$ 
The magnitude of the association between CVD, cardiovascular risk factors and COPD has been explored in systematic reviews and meta-analysis. Thus, the prevalence of cardiovascular comorbidities is twofold increase in COPD patients with an odds ratio (OR) between 1.90 and 2.46. ${ }^{5,6}$ Two observational multicenter studies carried out in patients hospitalized due to COPD exacerbations in Spain, also showed elevated cardiovascular comorbidity, with a prevalence of $32.8 \%$ for chronic heart failure, $20.8 \%$ for ischemic heart disease and $16.8 \%$ for peripheral vascular disease, among others. ${ }^{7,8}$ The presence of these diseases was related to a lower survival at 3-months after discharge. ${ }^{8}$ In outpatients with stable COPD, the ECLIPSE study shows that CVD was related to greater 3-years mortality after their adjustment for age, gender and smoking history. ${ }^{9}$ The relevance of CVD and other comorbidities in COPD patients for mortality and severe exacerbations can be assessed by the CODEX index. ${ }^{10}$ The comorbidity, obstruction, dyspnoea, exacerbations (CODEX) index is a multicomponent scale designed to predict the risk of readmissions and mortality at 1 year for patients hospitalized for COPD. The index includes the comorbidities (Charlson index adjusted by age), the degree of airway obstruction (post-bronchodilator $\mathrm{FEV}_{1} \%$ ), dyspnea (stratified according to the modified Medical Research Council scale), and severe exacerbations in the previous year. It has shown to be useful at predicting survival and readmission after hospital discharge for a COPD exacerbation, with a prognostic capacity superior to other previously published indices. ${ }^{11,12}$

Since COPD is related with an increased risk of cardiovascular events, especially in the more advanced phase of the disease and in patients with exacerbations, assessment of the overall cardiovascular risk in these patients seems essential, and similar therapeutic target levels for glycosylated hemoglobin, low-density lipoprotein cholesterol (LDL-C), blood pressure, dietary interventions and adapted physical activity recommended by clinical practice guidelines for patients at high cardiovascular risk should be achieved. ${ }^{13}$

We aimed to describe salient findings of major cardiovascular comorbidities associated with COPD based on the current best evidence from the recent medical literature (published from 2015), which, on the other hand, will contribute to reinforce recommendations of a recent Spanish consensus on the management of COPD according to the CODEX index. ${ }^{10}$

\section{Links Between COPD and CVD Smoking}

COPD and CVD share a number of common risk factors, including but not limited to smoking, the presence of which undoubtedly forms part of the explanation for the coexistence of COPD and CVD. Smoking and COPD are inextricably linked, while smoking is also a wellestablished major risk factor for COPD, atherosclerotic disease, coronary heart disease, heart failure, and peripheral artery disease.

\section{Aging}

Aging is closely related to the prevalence of chronic diseases, and some authors consider COPD as a disease of accelerated lung aging. ${ }^{14,15}$ Aging lung is characterized by several physiological and structural changes similar to those found in COPD. ${ }^{16,17}$ Several common pathways related to aging for COPD and CVD have been described, including oxidative stress, inflammation, telomere shortening, and genetic overlap, among others. ${ }^{15,18-21}$

\section{Incomplete Lung Development}

More recently, several cohort studies have shown that between $4 \%$ and $12 \%$ of the persons in the general population do not reach the peak lung considered normal for their age and sex in early adulthood. Many of those persons have chronic airflow limitation later in life, even though the rate of decline in $\mathrm{FEV}_{1}$ after its peak is similar to the observed in people without disease. ${ }^{22}$ Interestingly, these patients developed cardiovascular disease a decade before that the individuals with normal lung function in early adulthood, in an independent form to cumulative smoking exposure. ${ }^{23}$

\section{Classical Cardiovascular Risk Factors}

COPD patients have a higher prevalence of diabetes mellitus, arterial hypertension, dyslipidemia, and metabolic syndrome. Hypertension is the most common concurrent disorder among patients with $\mathrm{COPD}{ }^{24}$ In the metaanalysis of Chen et al, ${ }^{6}$ COPD patients showed an increased risk of arterial hypertension (odds ratio, OR $1.33,95 \%$ CI 1.33-1.56). In a recent cross-sectional study, COPD was independently associated with hypertension (OR 1.71, 95\% CI 1.37-2.13) after adjusting for age, obesity, smoking status, diabetes, and metabolic syndrome. ${ }^{25}$ In another study, half of the patients hospitalized for COPD exacerbation had a previous diagnosis of 
hypertension. ${ }^{8}$ The prevalence of diabetes is also increasing in COPD, particularly in patients with worse lung function. ${ }^{26}$ Similarly, the metabolic syndrome is more frequent in COPD patients, with an OR of 1.47 (95\% CI 1.09-1.88). ${ }^{27}$ Hypertension, abdominal obesity and hyperglycemia are the most prevalent components of the metabolic syndrome in COPD patients. ${ }^{28}$ Hospitalized COPD patients with metabolic syndrome had more dyspnea and higher comorbidity, including CVD. ${ }^{29}$

\section{Systemic Inflammation}

Low-grade systemic inflammation is considered a hallmark of the pathogenesis in COPD and one of the key mechanisms that may be responsible for the systemic effects on distant tissues and the increased rate of comorbidities, including cardiovascular comorbidity. ${ }^{30}$ The extent of the inflammatory reaction is correlated with the severity of the disease and is associated with an increase in mortality. ${ }^{31}$ Also, low-grade systemic inflammation plays an increasingly recognized role in the pathogenesis of atherosclerosis. The most validated markers of systemic inflammation in COPD are fibrinogen and high-sensitivity C-reactive protein, and both are also useful predictors of cardiovascular events in COPD and general population. ${ }^{32,33}$

\section{Thrombocytosis and Platelet Reactivity}

Thrombocytosis and platelet aggregation are especially relevant during COPD exacerbation and in the following weeks after the episode. The data from the SUMMIT study conducted in more than 16,000 patients with COPD and moderate obstruction $\left(\mathrm{FEV}_{1}>50 \%\right)$ confirm that the risk of presenting a cardiovascular event after hospitalization for COPD increases almost 10 times in the first month (hazard ratio, HR 9.9, 95\% CI 6.6-14.9) compared to patients without exacerbations. ${ }^{34}$ Between the mechanisms that may account for this increased CVD risk during exacerbations, thrombocytosis and platelet aggregation can be a suggestive explanation. A study carried out in 1343 patients hospitalized for COPD exacerbation shows that thrombocytosis during admission was related to both 1-year mortality and in-hospital mortality. ${ }^{35}$ In this study, antiplatelet therapy was associated with significantly lower 1-year mortality. In another prospective study, platelet reactivity was shown to be increased during COPD exacerbations. ${ }^{36}$ Similarly, in patients with an acute coronary event diagnosed by percutaneous coronary intervention (PCI), platelet reactivity was significantly higher at the time of PCI in COPD patients and remains increased 1-month after the procedure. ${ }^{37} \mathrm{~A}$ systematic review and meta-analysis suggested that antiplatelet therapy reduces all causes of mortality in COPD patients. ${ }^{38}$

\section{Serum Biomarkers}

Multiple serum biomarkers have been related to the progression of the disease and mortality in large cohorts of COPD patients. ${ }^{39}$ Some of them, such as surfactant proteinD (SPD), ClubCell-16 (CC-16), and desmosine, are generated in the lungs and are increased in the serum of COPD patients, relating to a higher risk of cardiovascular events. SPD is predominantly produced in type 2 pneumocytes and is also expressed in the endothelium of the cardiovascular system, acting as a mediator on inflammatory signals. ${ }^{40}$ SPD has been associated in longitudinal studies with an increase of cardiovascular mortality in COPD patients. ${ }^{41}$ CC-16 are secreted mainly in the terminal portion of the bronchioles and their plasma levels are inversely associated with an increase in mortality in COPD. ${ }^{42}$ Desmosine is a specific marker of elastin degradation and is increased in patients with COPD, especially in those with emphysema. Plasma desmosine levels in COPD patients are higher in those with a history of cardiovascular disease and are associated with higher levels of coronary calcification measured by $\mathrm{CT}$ and 3-years mortality. ${ }^{43}$

Especially relevant are biomarkers of myocardial damage in COPD. A cohort study performed in more than 15,000 patients with available data of spirometric and echocardiographic measures, alongside serum levels of ultrasensitive troponin and NT pro-BNP levels, has demonstrated the close relationship between lung function and these biomarkers. ${ }^{44}$ On the other hand, several studies have shown that the elevation of troponin and pro-BNP, both during exacerbations and in the stable phase, is associated with higher mortality in patients with COPD, even in those without known ischemic heart disease or heart failure. ${ }^{45,46}$ In a cohort study performed in patients with severe exacerbations of COPD and elevated levels of troponins at admission, coronary angiography performed 72 hours after hospitalization showed significant coronary stenosis $(>50 \%)$ in major coronary arteries in $67 \%$ of patients, of which $39 \%$ required percutaneous coronary intervention. ${ }^{47}$

\section{Arterial Stiffness}

Arterial stiffness is a predictor of mortality and cardiovascular events in patients with CVD and in the general population. ${ }^{48}$ Several studies and meta-analysis have shown that arterial stiffness is also increased in COPD 
patients in whom it has been related to the severity of the obstruction, systemic inflammation, and previous history of exacerbations. ${ }^{49}$ In a longitudinal study, arterial stiffness increase during COPD exacerbations jointly with serum troponin levels. ${ }^{50}$ Of note, arterial stiffness in COPD patients is related to serum levels of SPD, CC-16, and desmosine. ${ }^{42,43}$ Another study performed in patients with a coronary event diagnosed by coronary angiography and COPD diagnosed by spirometry confirmed that pulmonary function is closely related to arterial stiffness. ${ }^{51}$

\section{Heart Failure}

The combination of HF and COPD incur significant morbidity and mortality, and present major challenges to health-care providers. The reported prevalence of COPD in patients with HF varies largely (from $11 \%$ to $52 \%$ ) in North American patients and (from 9\% to 41\%) in European cohorts. ${ }^{52}$ Geographical variations largely relate to differences in population age structure and risk factor exposure, most notably smoking. On the other hand, cigarette smoking, the commonest cause of COPD, is associated with a substantial increased risk of HF. ${ }^{53}$ The prevalence of left ventricular systolic dysfunction (LVSD) in patients with COPD varies considerably between $10 \%$ and $46 \%$, with lower prevalences when excluding patients with coronary disease. ${ }^{54}$

\section{Systematic Reviews and Meta-Analysis}

In a systematic review of 25 studies published between 1990 and 2012 reporting cardiovascular comorbidity in patients with COPD (or vice versa), the adjusted risk ratio (RR) for the prevalence of $\mathrm{HF}$ in COPD ranged from 1.8 to 3.9 compared with patients without COPD, and the adjusted RR for hospitalization from 1.2 to $3.8 .^{55}$ In the meta-analysis of 29 datasets from 27 studies, a significant increase in the prevalence of HF in COPD was found in 14 datasets, yielding a pooled OR of 2.57 (95\% CI 1.90-3.47; $P<0.0001){ }^{6}$

\section{Primary Studies}

In a longitudinal study of COPD patients hospitalized with decompensated heart failure all 11 medical centers in central Massachusetts during four study years: 1995, 2000, 2002, and 2004, and followed through 2010 for determination of their vital status, COPD was associated with a $10 \%$ increase in 1-year mortality and a $40 \%$ increase in 5-year mortality. ${ }^{56}$ Moreover, the finding of undertreatment with $\beta$-blockers in patients with COPD despite temporal increases in use highlights a key area for quality improvement. In the Spanish consensus on the management of COPD according to the CODEX index, the panel agreed that patients with COPD and heart failure or ischemic heart disease have to be treated with $\beta$-blockers. ${ }^{10}$

In an analysis of the Spanish National Hospital Discharge Database (2001-2015), admissions for $\mathrm{HF}$ increased by $2.9 \%$ per year among men with COPD. ${ }^{57}$ In a study of costs associated with readmissions in patients with both HF and COPD, 10 practical tips to reduce readmissions were presented, including 1) diagnose the population accurately, 2) detect admissions for exacerbations early and consider risk stratification, 3) use specialist management in hospital, 4) modify the underlying disease substrate, 5) apply and intensify evidence-based therapies, 6) activate the patient and develop critical health behaviors, 7) setup feedback loops, 8) arrange an early follow-up appointment prior to discharge, 9) consider and address other comorbidities, and 10) consider ancillary support services at home. ${ }^{58}$ In a cross-sectional observational study carried out in Toronto, Canada, in which agreement between hospital and primary care on diagnostic labeling for COPD and heart failure was analyzed, COPD concordance was 34\% and $\mathrm{HF}$ concordance 33\%; moreover, $21-24 \%$ additional patients with COPD and $18-20 \%$ additional patients with HF did not have a label in either setting. ${ }^{59}$ This calls for the need for integrated disease management between the different physicians and nurses of these patients and between the hospital and primary care.

\section{Coronary Heart Disease}

In the last few years, many studies focused their attention on the relationship between COPD and coronary heart disease showing that these disorders are mutually influenced. The prevalence of coronary heart disease - a term that includes myocardial infarction (MI), angina, coronary artery disease, and ischemic heart disease (IHD) - ranged between $4.7 \%$ and $60 \%$ among patients with COPD, with an adjusted RR ranging from 0.7 to $6.8 .^{55}$ Also, in a robust meta-analysis of the risk of cardiovascular comorbidity in COPD, it was found that patients with COPD had consistent higher risks of coronary heart disease (OR 1.96, 95\% CI 1.51-2.30; $P<0.0001$ ), MI (OR 2.71, 95\% CI 1.69$4.35 ; P<0.0001$ ), and angina pectoris (OR 8.16, 95\% CI $3.08-21.59 ; P<0.0001){ }^{6}$ Indeed, COPD patients with IHD may have worse outcomes. The three-year followup of 4284 patients who received hospital treatment for coronary heart disease reported mortality rates of $21 \%$ for 
patients diagnosed with COPD versus $9 \%$ in those without COPD $(P<0.001) .{ }^{60}$ Likewise, patients with ST segment elevation $\mathrm{MI}$ and concomitant COPD are at greater risk for death and hospital readmissions due to cardiovascular causes (eg recurrent MI, HF, bleeding) than patients without COPD. ${ }^{61}$

\section{Systematic Reviews and Meta-Analysis}

In a systematic review and meta-analysis of 10 studies (7518 patients with COPD and 65,451 patients without COPD), a comparison of major adverse cardiac events (MACEs) and mortality following percutaneous coronary intervention revealed that in-hospital (OR 1.40, 95\% CI $1.19-1.65 ; P=0.001)$ and long-term MACEs (OR 1.58, $95 \%$ CI 1.38-1.81, $P=0.001$ ) were significantly higher in patients with COPD. ${ }^{62}$ Therefore, COPD should be considered a risk factor for the development of adverse clinical outcomes following coronary revascularization procedures. Regrettably, in all of these studies, definition of COPD is based in the clinical history or discharge codes, without spirometric confirmation and underdiagnosis of COPD in IHD is about $80 \% .{ }^{63-65}$ In a meta-analysis of 24 observational studies, an increased risk of MI associated with COPD (HR 1.72, 95\% CI 1.22-2.42) and with acute exacerbation of COPD (incidence rate ratio [IRR] 13.04, 95\% CI 1.71 to 99.7 ) was found. ${ }^{66}$ Therefore and from a clinical perspective, acute exacerbations of COPD represent periods of increased risk of MI.

\section{Primary Studies}

Recent studies have drawn attention to the economic burden of co-occurrence of COPD and IHD, particularly the high expenditure for medications involved with IHD pharmacotherapy in the course of COPD. ${ }^{67}$ In an analysis of German Statutory Health Insurance claims data based on 26,318 COPD patients with and 10,287 COPD patients without IHD, IHD was a substantial cost driver in COPD with the highest excess costs in the age group between 70 and 80 years. ${ }^{68}$

In a Sweden population-based COPD cohort, the presence of self-reported IHD was estimated in comparison with age- and sex-matched subjects without COPD. ${ }^{69}$ There was a significant association between self-reported IHD as well as probable ischemic ECG-changes and COPD disease severity assessed by spirometry. Also, in the same Sweden cohort, ischemic ECG changes were associated with an increased risk for death (RR 2.36, 95\% CI 1.45-3.85) when compared with subjects with normal lung function, even after adjusting for common confounders (RR 1.65, 95\% CI 0.94-2.90). The risk of death also persisted after adjustment for COPD severity. ${ }^{70}$ Underuse of beta-blockers, statins, and antiplatelet drugs is frequent in patients with IHD and COPD, and possibly contributes to a worse prognosis. ${ }^{71,72}$ Nevertheless, even in patients with IHD adequately treated, the presence of COPD confirmed by spirometry is associated with an increase in cardiovascular events and mortality during the follow-up. ${ }^{63,73}$

\section{Peripheral Arterial Occlusive Disease}

Peripheral arterial occlusive disease (PAOD) is the clinical expression of the artery obstruction, usually of the lower limbs, secondary to atherosclerotic disease. Tobacco smoking is the strongest risk factor for both COPD and PAOD. Intermittent claudication is the most characteristic symptom of PAOD, although more of the half of the patients can be asymptomatic. Since intermittent claudication is initially manifested with physical effort, and patients with severe COPD are often impaired for dyspnea at the exercise, the diagnosis of PAOD is frequently delayed. A simple method for evaluating PAOD is the ankle-brachial index (ABI). The $\mathrm{ABI}$ is also a strong marker of generalized atherosclerosis and $\mathrm{CV}$ risk. An ABI $<0.90$ is strongly suggestive of PAOD, and is associated on average with a twofold to threefold increased risk of total and CV death. ${ }^{74}$

\section{Systematic Reviews and Meta-Analysis}

In the meta-analysis of Chen et $\mathrm{al}^{6}$ based on seven datasets, the pooled risk for the prevalence of PAOD in COPD patients was 2.35 (95\% CI 1.48-3.74; $P=0.003)$. These data are based on population studies and therefore, do not include subclinical PAOD. In another recent systematic review, based on six studies with diagnostic confirmation of PAOD with $\mathrm{ABI}$ and COPD with spirometry, COPD patients with PAOD were more frequently males with tobacco history, hypertension, dyslipidemia, higher values of fibrinogen and C-reactive protein, lower values of $\mathrm{FEV}_{1} \%$, and higher dyspnea levels. $^{75}$

\section{Primary Studies}

A number of primary studies of the effect of PAOD in COPD patients have been published and included in systematic reviews and meta-analysis, ${ }^{6,75}$ but a recent study sought to investigate the incidence of PAOD among COPD patients in Taiwan using a national database reports interesting data. In this study, 51,869 COPD patients were collected from the 
National Health Insurance Research Database of Taiwan from 1996 to 2010 and compared with the same number of controls without COPD. The incidence of PAOD was higher in the COPD group than in the non-COPD group (HR 1.23, 95\% CI 1.17-1.29). ${ }^{76}$ Also, the risk of PAOD increased with the number of comorbidities, with HRs increasing from 3.7 to 4.9 when the number of COPD comorbidities increased from $\geq 1$ to $\geq 3{ }^{76}$

\section{Pulmonary Hypertension}

Pulmonary hypertension (PHT) is a clinically relevant problem in COPD due to its high prevalence and the impact that it exerts on morbidity and mortality. Hypoxia is a critical precipitant in the pathogenesis of COPD-associated PHT and, at least in part, explains the correlation between the severity of COPD and the development of PHT. COPD patients with coexisting sleep apnea have a higher risk of developing pulmonary hypertension. ${ }^{77}$ Hypoxia induces pulmonary vasoconstriction and pulmonary vascular remodeling in the form of intimal thickening and muscularization of arterioles, thereby increasing pulmonary vascular resistance. Dynamic pulmonary hyperinflation, endothelial dysfunction, polycythemia, inflammation, and parenchymal destruction have also been implicated in the pathogenesis of increased pulmonary vascular resistance in patients with COPD. ${ }^{4} \mathrm{PHT}$ is present in more than $25 \%$ and $50 \%$ of the patients with moderate-to-severe COPD, and the 5-year survival of patients suffering PHT is almost half of patients without PHT (36\% and $62 \%$, respectively). ${ }^{78,79}$ Furthermore, signs of elevated pulmonary artery pressure are associated with more frequent exacerbation episodes. In the meta-analysis of Chen et al of the risk of cardiovascular comorbidity in COPD, the odds of patients with COPD having diseases of pulmonary circulation were 5.14 times higher as compared to matched controls without COPD. ${ }^{6}$ A small proportion of COPD patients may present with "out-of-proportion" pulmonary hypertension, in these patients concomitant cardiac disorders, chronic pulmonary thromboembolic disease or a concurrent form of primary pulmonary hypertension should be considered. ${ }^{80}$

\section{Systematic Reviews and Meta-Analysis}

Angiotensin-converting enzyme (ACE) gene I/D polymorphism has been studied in relation to the susceptibility to COPD and COPD with PHT with inconclusive results. A systematic review with meta-analysis of 15 studies (2635 participants, 288 participants of the PHT subgroup) showed that ACE gene polymorphism, particularly the homozygote variant, was associated with an increased risk of PHT in Asian COPD patients. ${ }^{79}$ These results, however, should be validated in larger sample populations and in more ethnicities.

\section{Primary Studies}

Patients with severe PHT associated with COPD present a poor outcome. Studies in a limited number of patients with severe PHT and moderate-to-severe COPD have shown that specific pulmonary arterial hypertension therapy can improve pulmonary hemodynamic parameters. ${ }^{81}$

\section{Cerebrovascular Disease}

In contrast to the evidence suggesting that COPD increases the risk of heart disease, studies conducted on the risk of cerebrovascular disease among COPD patients have generated conflicting results. In a systematic review of 25 studies, the adjusted RR for stroke ranged between 1.1 and 1.6, with apparently an increasing trend by increasing grade or airflow limitation. ${ }^{55}$ However, in the systematic review of Chen et al, the current evidence on stroke risk among COPD patients was limited by a dependence on cross-sectional data, which precluded a firm establishment of causality between COPD and stroke. ${ }^{6}$

\section{Systematic Reviews and Meta-Analysis}

A recent systematic review and meta-analysis of eight studies, mostly conducted in Western countries and in patients with newly developed stroke, a significantly increased risk of stroke was observed among COPD patients (HR 1.30; 95\% CI 1.18-1.43; $P<0.001$ ). ${ }^{82}$ The association between COPD and stroke risk remained robust in subgroup analyses by stroke subtype, study quality, and adjustment for socioeconomic status.

\section{Primary Studies}

Patients with COPD have a higher risk of stroke than the general population, and chronic inflammation associated with COPD is thought to contribute to this risk. Exacerbations of COPD are associated with a rise in inflammation, suggesting that there may be an association between exacerbation frequency and the risk of stroke. In a study using the UK Clinical Practice Research Datalink, COPD patients with a first stroke between January 2004 and December 2013 were identified as cases and matched on age, sex, and general practice to controls with COPD but without a stroke (6441 cases and 19,323 controls). ${ }^{83}$ There was no evidence that frequent exacerbators $(\geq 2$ 
episodes in the previous year) had an increased stroke risk compared to infrequent exacerbators $(\leq 1$ episode) (OR $0.95,95 \%$ CI $0-89-1.01)$. These results suggest that exacerbation frequency is unlikely to be the reason for increased stroke risk among COPD patients. However, in a study using Taiwan's National Health Insurance Research Database, a comparison cohort of 1918 adults with COPD exacerbations, 3836 adults with COPD no exacerbations, and 7672 adults without COPD who were frequency matched by age and sex, were selected. ${ }^{84}$ Patients with exacerbations had increased stroke incidence (adjusted HR 1.28, 95\% CI 1.03-1.59) and post-stroke mortality (OR 1.34, 95\% CI 1.20-1.52) and complications, including, epilepsy and pneumonia. In a study that assessed the prevalence of COPD among hospitalized stroke patients in the National Inpatient Sample, a nationally representative dataset of US hospital admissions between January 2004 and December 2009, 12\% of the hospitalized stroke patients have COPD. The crude and age-adjusted in-hospital mortality rates for these patients were $6.3 \%$ (95\% CI $6.14-6.53 \%)$ and $6.0 \%$ (95\% CI $4.05-7.94 \%$ ), respectively, with greater risks of mortality seen among those with intracerebral hemorrhage. This study shows that the presence of COPD is an independent risk factor for early mortality in stroke patients. ${ }^{85}$

\section{Concluding Remarks}

Among the long list of comorbid conditions seen in patients with COPD, cardiovascular diseases rank not only among the most common but also as associated with an increased risk of death. However, despite the broad acceptance of prognostic significance of these diseases, including chronic heart failure, coronary artery disease, peripheral arterial occlusive disease and cerebrovascular events as shown in the present article, there remains widespread under-recognition and undertreatment of comorbid cardiovascular diseases in COPD population. The reasons for this are unclear but lack of specific evidence-based guidelines for the management of cardiovascular disorders and cardiovascular risk factors in people with COPD may be an important contributory factor. Optimizing the treatment of COPD and cardiovascular diseases, including the prevention of cardiovascular risk can improve the prognosis of these patients. ${ }^{86}$

\section{Acknowledgments}

The authors would like to thank Chiesi for their continued and constant support for this project, to GOC
Networking team for their support and collaboration in the development of this document, and Marta Pulido, $\mathrm{MD}, \mathrm{PhD}$, for editing the manuscript and editorial assistance.

\section{Author Contributions}

All authors made substantial contributions to conception and design, acquisition of data, or analysis and interpretation of data; took part in drafting the article or revising it critically for important intellectual content; gave final approval of the version to be published; and agree to be accountable for all aspects of the work.

\section{Funding}

This work was supported by a grant from Chiesi.

\section{Disclosure}

Pere Almagro has received speaker or consulting fees from Chiesi, AstraZeneca, Boehringer-Ingelheim, GlaxoSmithKline, Laboratorios Esteve, Rovi, Menarini y Novartis unrelated to this manuscript.

Ramon Boixeda has received speaker or consulting fees from Chiesi, Boehringer-Ingelheim, GlaxoSmithKline, Rovi, and Novartis unrelated to this manuscript.

Jesús Díez-Manglano has received speaker or consulting fees from Bayer, Bristol-Myers-Squibb, Chiesi, and Novartis unrelated to this manuscript.

María Gómez-Antúnez has received speaker or consulting fees from Chiesi, AstraZeneca, Rovi, and Novartis unrelated to this manuscript.

Francisco López-García has received speaker or consulting fees from Chiesi, AstraZeneca, BoehringerIngelheim, GlaxoSmithKline and Novartis unrelated to this manuscript.

Jesús Recio has received speaker or consulting fees from Chiesi, Boehringer-Ingelheim, Laboratorios Ferrer and Novartis unrelated to this manuscript. The authors report no other conflicts of interest in this work.

\section{References}

1. (WHO) 1. World Health Organization. Chronic obstructive pulmonary disease (COPD). 1 December 2017. Key facts. Available from: https:// www.who.int/news-room/fact-sheets/detail/chronic-obstructivepulmonary-disease-(copd). Accessed July 22, 2019.

2. Vos T, Allen C, Arora M, et al. Global, regional, and national incidence, prevalence, and years lived with disability for 310 diseases and injuries, 1990-2015: a systematic analysis for the Global Burden of Disease Study 2015. Lancet. 2016;388(10053):1545-1602. doi:10.10 16/S0140-6736(16)31678-6 
3. Vanfleteren LEGW, Spruit MA, Groenen M, et al. Clusters of comorbidities based on validated objective measurements and systemic inflammation in patients with chronic obstructive pulmonary disease. Am J Respir Crit Care Med. 2013;187(7):728-735. doi:10.1164/rccm.201209-1665OC

4. Smith MC, Wrobel JP. Epidemiology and clinical impact of major comorbidities in patients with COPD. Int J COPD. 2014;9:871-888. doi:10.2147/COPD.S49621

5. Yin HL, Yin SQ, Lin QY, Xu Y, Xu HW, Liu T. Prevalence of comorbidities in chronic obstructive pulmonary disease patients. Med. 2017;96(19):e6836. doi:10.1097/MD.0000000000006836

6. Chen W, Thomas J, Sadatsafavi M, FitzGerald JM. Risk of cardiovascular comorbidity in patients with chronic obstructive pulmonary disease: a systematic review and meta-analysis. Lancet Respir Med. 2015;3(8):631-639. doi:10.1016/S2213-2600(15)00241-6

7. Almagro P, López García F, Cabrera FJ, et al. Study of the comorbidities in hospitalized patients due to decompensated chronic obstructive pulmonary disease attended in the Internal Medicine Services. ECCO Study. Rev Clin Esp. 2010;210(3):101-108. doi:10. 1016/j.rce.2009.12.002

8. Almagro P, Cabrera FJ, Diez J, et al. Comorbidities and short-term prognosis in patients hospitalized for acute exacerbation of COPD: the EPOC en servicios de medicina interna (ESMI) study. Chest. 2012;142(5):1126-1133. doi:10.1378/chest.11-2413

9. Miller J, Edwards LD, Agustí A, et al. Comorbidity, systemic inflammation and outcomes in the ECLIPSE cohort. Respir Med. 2013;107 (9):1376-1384. doi:10.1016/j.rmed.2013.05.001

10. Boixeda R, Díez-Manglano J, Gómez-Antúnez M, López-García F, Recio J, Almagro P. Consensus for managing patients with chronic obstructive pulmonary disease according to the CODEX index. Rev Clin Esp. 2019;219(9):494-504. doi:10.1016/j.rce.2019.03.006

11. Almagro P, Soriano JBJB, Cabrera FJFJ, et al. Short- and medium-term prognosis in patients hospitalized for COPD exacerbation: the CODEX index. Chest. 2014;145(5):972-980. doi:10.1378/chest.13-1328

12. Deng D, Zhou A, Ping C, Shuang Q. CODEXS: a new multidimensional index to better predict frequent COPD exacerbators with inclusion of depression score. Int J Chron Obstruct Pulmon Dis. 2020;2020:249-259. doi:10.2147/COPD.S237545

13. Arnett DKBR, Albert MA, Buroker AB, et al. 2019 ACC/AHA guideline on the primary prevention of cardiovascular disease: executive summary: a report of the American College of Cardiology/ American Heart Association Task Force on Clinical Practice Guidelines. Circulation. 2019;74(10):e177-e232.

14. Kennedy BK, Berger SL, Brunet A, et al. Geroscience: linking aging to chronic disease. Cell. 2014;159(4):709-713. doi:10.1016/j.cell. 2014.10.039

15. Ito K, Barnes PJ. COPD as a disease of accelerated lung aging. Chest. 2009;135(1):173-180. doi:10.1378/chest.08-1419

16. Brandsma C, Van den Berge M, Hackett T, Brusselle G, Timens W. Recent advances in chronic obstructive pulmonary disease pathogenesis: from disease mechanisms to precision medicine. J Pathol. 2019. doi:10.1002/path.5364

17. Steenman M, Lande G. Cardiac aging and heart disease in humans. Biophys Rev. 2017;9(2):131-137. doi:10.1007/s12551-017-0255-9

18. Fyhrquist F, Saijonmaa O, Strandberg T. The roles of senescence and telomere shortening in cardiovascular disease. Nat Rev Cardiol. 2013;10(5):274-283. doi:10.1038/nrcardio.2013.30

19. North BJ, Sinclair DA. The intersection between aging and cardiovascular disease. Circ Res. 2012;110(8):1097-1108. doi:10.1161/ CIRCRESAHA.111.246876

20. Liguori I, Russo G, Curcio F, et al. Oxidative stress, aging, and diseases. Clin Interv Aging. 2018;13:757-772. doi:10.2147/CIA.S158513

21. Zhu Z, Wang X, Li X, et al. Genetic overlap of chronic obstructive pulmonary disease and cardiovascular disease-related traits: a large-scale genome-wide cross-trait analysis. Respir Res. 2019;20 (1):64. doi:10.1186/s12931-019-1036-8
22. Lange $\mathrm{P}$, Celli B, Agustí A, et al. Lung-function trajectories leading to chronic obstructive pulmonary disease. $N$ Engl J Med. 2015;373 (2):111-122. doi:10.1056/NEJMoa1411532

23. Agustí A, Noell G, Brugada J, Faner R. Lung function in early adulthood and health in later life: a transgenerational cohort analysis. Lancet Respir Med. 2017;5(12):935-945. doi:10.1016/S22 13-2600(17)30434-4

24. Finks SW, Rumbak MJ, Self TH. Treating hypertension in chronic obstructive pulmonary disease. $N$ Engl J Med. 2020;382(4):353-363. doi:10.1056/NEJMra1805377

25. Kim SH, Park JH, Lee JK, Heo EY, Kim DK, Chung HS. Chronic obstructive pulmonary disease is independently associated with hypertension in men. Med. 2017;96(19):e6826. doi:10.1097/MD.000 0000000006826

26. Mannino DM, Thorn D, Swensen A, Holguin F. Prevalence and outcomes of diabetes, hypertension and cardiovascular disease in COPD. Eur Respir J. 2008;32(4):962-969. doi:10.1183/09031936.00012408

27. Lam K-BH, Jordan RE, Jiang CQ, et al. Airflow obstruction and metabolic syndrome: the Guangzhou Biobank Cohort Study. Eur Respir J. 2010;35(2):317-323. doi:10.1183/09031936.00024709

28. Cebron Lipovec N, Beijers RJHCG, van den Borst B, Doehner W, Lainscak M, Schols AMWJ. The Prevalence of metabolic syndrome in chronic obstructive pulmonary disease: a systematic review. COPD J Chronic Obstr Pulm Dis. 2016;13(3):399-406. doi:10.31 09/15412555.2016.1140732

29. Diez-Manglano J, Barquero-Romero J, Almagro P, et al. COPD patients with and without metabolic syndrome: clinical and functional differences. Intern Emerg Med. 2014;9(4):419-425. doi:10.1007/s11 739-013-0945-7

30. Sinden NJ, Stockley RA. Systemic inflammation and comorbidity in COPD: a result of "overspill" of inflammatory mediators from the lungs? Review of the evidence. Thorax. 2010;65(10):930-936. doi:10.1136/thx.2009.130260

31. Eapen MS, Myers S, Walters EH, Sohal SS. Airway inflammation in chronic obstructive pulmonary disease (COPD): a true paradox. Expert Rev Respir Med. 2017;11(10):827-839. doi:10.1080/17476 348.2017.1360769

32. Global Initiative for Chronic Obstructive Pulmonary Disease. 2020 GOLD Reports. 2020 Global strategy for prevention, diagnosis and management of COPD. Available from: https://goldcopd.org/goldreports/. Accessed February 23, 2020.

33. Kaptoge S, Di Angelantonio E, et al.; Emerging Risk Factors Collaboration. C-reactive protein, fibrinogen, and cardiovascular disease prediction. $N$ Engl J Med. 2012;367(14):1310-1320. doi:10.10 56/NEJMoa1107477

34. Kunisaki KM, Dransfield MT, Anderson JA, et al. Exacerbations of chronic obstructive pulmonary disease and cardiac events. a post hoc cohort analysis from the SUMMIT randomized clinical trial. Am J Respir Crit Care Med. 2018;198(1):51-57. doi:10.1164/rccm.201711-2239OC

35. Harrison MT, Short P, Williamson PA, Singanayagam A, Chalmers JD, Schembri S. Thrombocytosis is associated with increased short and long term mortality after exacerbation of chronic obstructive pulmonary disease: a role for antiplatelet therapy? Thorax. 2014;69(7):609-615. doi:10.1136/thoraxjnl-2013-203996

36. Muñoz-Esquerre M, Ferreiro JL, Huertas D, et al. Impact of acute exacerbations on platelet reactivity in chronic obstructive pulmonary disease patients. Int $J$ COPD. 2018;13:141-148. doi:10.2147/COPD. S152660

37. Campo G, Pavasini R, Pollina A, Tebaldi M, Ferrari R. On-treatment platelet reactivity in patients with chronic obstructive pulmonary disease undergoing percutaneous coronary intervention. Thorax. 2014;69(1):80-81. doi:10.1136/thoraxjnl-2013-203608

38. Pavasini R, Biscaglia S, d'Ascenzo F, et al. Antiplatelet treatment reduces all-cause mortality in COPD patients: a systematic review and meta-analysis. COPD. 2015;2555(January2016):1-6. doi:10.31 $09 / 15412555.2015 .1099620$ 
39. Zemans RL, Jacobson S, Keene J, et al. Multiple biomarkers predict disease severity, progression and mortality in COPD. Respir Res. 2017;18(1):117. doi:10.1186/s12931-017-0597-7

40. Sorensen GL. Surfactant protein D in respiratory and non-respiratory diseases. Front Med. 2018;5:18. doi:10.3389/fmed.2018.00018

41. Hill J, Heslop C, Man SFP, et al. Circulating surfactant protein-D and the risk of cardiovascular morbidity and mortality. Eur Heart J. 2011;32(15):1918-1925. doi:10.1093/eurheartj/ehr124

42. Labonté LE, Bourbeau J, Daskalopoulou SS, et al. Club cell-16 and RelB as novel determinants of arterial stiffness in exacerbating COPD patients. PLoS One. 2016;11(2):e0149974. doi:10.1371/journal.pone.0149974

43. Rabinovich RA, Miller BE, Wrobel K, et al. Circulating desmosine levels do not predict emphysema progression but are associated with cardiovascular risk and mortality in COPD. Eur Respir J. 2016;47 (5):1365-1373. doi:10.1183/13993003.01824-2015

44. Baum C, Ojeda FM, Wild PS, et al. Subclinical impairment of lung function is related to mild cardiac dysfunction and manifest heart failure in the general population. Int J Cardiol. 2016;218:298-304. doi:10.1016/j.ijcard.2016.05.034

45. Pavasini R, Tavazzi G, Biscaglia S, et al. Amino terminal pro brain natriuretic peptide predicts all-cause mortality in patients with chronic obstructive pulmonary disease: systematic review and meta-analysis. Chron Respir Dis. 2017;14(2):117-126. doi:10.1177/ 1479972316674393

46. Pavasini R, D’Ascenzo F, Campo G, et al. Cardiac troponin elevation predicts all-cause mortality in patients with acute exacerbation of chronic obstructive pulmonary disease: systematic review and meta-analysis. Int J Cardiol. 2015;191:187-193. doi:10.1016/j.ijcard. 2015.05.006

47. Pizarro C, Herweg-Steffens N, Buchenroth M, et al. Invasive coronary angiography in patients with acute exacerbated COPD and elevated plasma troponin. Int $J$ Chron Obstruct Pulmon Dis. 2016;11:2081-2089. doi:10.2147/COPD.S110746

48. Ben-Shlomo Y, Spears M, Boustred C, et al. Aortic pulse wave velocity improves cardiovascular event prediction: an individual participant meta-analysis of prospective observational data from 17,635 subjects. J Am Coll Cardiol. 2014;63(7):636-646. doi:10.1016/j. jacc.2013.09.063

49. Fisk M, McEniery CM, Gale N, et al. Surrogate markers of cardiovascular risk and chronic obstructive pulmonary disease. Hypertension 2018;71(3):499-506. doi:10.1161/HYPERTENSIONAHA.117.10151

50. Patel ARC, Kowlessar BS, Donaldson GC, et al. Cardiovascular risk, myocardial injury, and exacerbations of chronic obstructive pulmonary disease. Am J Respir Crit Care Med. 2013;188(9):1091-1099. doi:10.1164/rccm.201306-11700C

51. Almagro P, Acosta E, Navarro A, Murillo MF, Valdivielso S, de la Sierra A. Study of arterial stiffness in patients with an acute coronary event and chronic obstructive pulmonary disease confirmed by spirometry. Rev Clin Esp. 2019;219(5):251-255. doi:10.1016/j. rce.2018.08.007

52. Hawkins NM, Petrie MC, Jhund PS, Chalmers GW, Dunn FG, McMurray JJV. Heart failure and chronic obstructive pulmonary disease: diagnostic pitfalls and epidemiology. Eur J Heart Fail. 2009;11(2):130-139. doi:10.1093/eurjhf/hfn013

53. He J, Ogden LG, Bazzano LA, Vupputuri S, Loria C, Whelton PK. Risk factors for congestive heart failure in US men and women: NHANES I epidemiologic follow-up study. Arch Intern Med. 2001;161(7):996-1002. doi:10.1001/archinte.161.7.996

54. Rutten FH, Cramer M-JM, Lammers J-WJ, Grobbee DE, Hoes AW. Heart failure and chronic obstructive pulmonary disease: an ignored combination? Eur J Heart Fail. 2006;8(7):706-711. doi:10.1016/j. ejheart.2006.01.010

55. Müllerova H, Agusti A, Erqou S, Mapel DW. Cardiovascular comorbidity in COPD: systematic literature review. Chest. 2013;144 (4):1163-1178. doi:10.1378/chest.12-2847
56. Fisher KA, Stefan MS, Darling C, Lessard D, Goldberg RJ. Impact of COPD on the mortality and treatment of patients hospitalized with acute decompensated heart failure. The worcester heart failure study. Chest. 2015;147(3):637-645. doi:10.1378/chest.14-0607

57. de Miguel-díez J, López-de-Andrés A, Hernández-Barrera V, et al. Influence of COPD on outcomes of patients hospitalized with heart failure: analysis of the Spanish National Hospital Discharge Database (2001-2015). Int J Cardiol. 2018;269:213-219. doi:10.1016/j.ijcard. 2018.07.067

58. Kalhan R, Mutharasan RK. Reducing readmissions in patients with both heart failure and COPD. Chest. 2018;154(5):1230-1238. doi:10.1016/j.chest.2018.06.001

59. Greiver M, Sullivan F, Kalia S, et al. Agreement between hospital and primary care on diagnostic labeling for COPD and heart failure in Toronto, Canada: a cross-sectional observational study. Npj Prim Care Respir Med. 2018;28(1):9. doi:10.1038/s41533-018-0076-8

60. Berger JS, Sanborn TA, Sherman W, Brown DL. Effect of chronic obstructive pulmonary disease on survival of patients with coronary heart disease having percutaneous coronary intervention. Am J Cardiol. 2004;94(5):649-651. doi:10.1016/j.amjcard.2004.05.034

61. Campo G, Guastaroba P, Marzocchi A, et al. Impact of COPD on long-term outcome after ST-segment elevation myocardial infarction receiving primary percutaneous coronary intervention. Chest. 2013;144(3):750-757. doi:10.1378/chest.12-2313

62. Bundhun PK, Gupta C, Xu GM. Major adverse cardiac events and mortality in chronic obstructive pulmonary disease following percutaneous coronary intervention: a systematic review and meta-analysis. BMC Cardiovasc Disord. 2017;17(1):1-13. doi:10.1186/s12872-0170622-2

63. Almagro P, Lapuente A, Pareja J, et al. Underdiagnosis and prognosis of chronic obstructive pulmonary disease after percutaneous coronary intervention: a prospective study. Int J Chron Obstruct Pulmon Dis. 2015;10:1353-1361. doi:10.2147/COPD.S84482

64. Franssen FME, Soriano JB, Roche N, et al. Lung Function abnormalities in smokers with ischemic heart disease. Am J Respir Crit Care Med. 2016;194(5):568-576. doi:10.1164/rccm.201512-2480OC

65. Soriano JB, Rigo F, Guerrero D, et al. High prevalence of undiagnosed airflow limitation in patients with cardiovascular disease. Chest. 2010;137(2):333-340. doi:10.1378/chest.09-1264

66. Rothnie KJ, Yan R, Smeeth L, Quint JK. Risk of myocardial infarction (MI) and death following MI in people with chronic obstructive pulmonary disease (COPD): a systematic review and meta-analysis. BMJ Open. 2015;5(9):e007824. doi:10.1136/bmjopen-2015-007824

67. Rysiak E, Prokop I, Zaręba I, Mróz RM. Estimates of medication expenditure for ischemic heart disease accompanying chronic obstructive pulmonary disease. Adv Exp Med Biol. 2018;1114: 49-55. doi:10.1007/55842018 201

68. Schwarzkopf L, Wacker M, Ertl J, Hapfelmeier J, Larisch K, Leidl R. Impact of chronic ischemic heart disease on the health care costs of COPD patients - An analysis of German claims data. Respir Med. 2016;118:112-118. doi:10.1016/j.rmed.2016.08.001

69. Nilsson U, Johansson B, Eriksson B, Blomberg A, Lundbäck B, Lindberg A. Ischemic heart disease among subjects with and without chronic obstructive pulmonary disease-ECG-findings in a populationbased cohort study. BMC Pulm Med. 2015;15(1):156. doi:10.1186/ s12890-015-0149-1

70. Nilsson U, Blomberg A, Johansson B, Backman H, Eriksson B, Lindberg A. Ischemic ECG abnormalities are associated with an increased risk for death among subjects with COPD, also among those without known heart disease. Int J Chron Obstruct Pulmon Dis. 2017;12:2507-2514. doi:10.2147/COPD.S136404

71. Rasmussen DB, Bodtger U, Lamberts M, et al. Beta-blocker, aspirin, and statin usage after first-time myocardial infarction in patients with chronic obstructive pulmonary disease: a nationwide analysis from 1995 to 2015 in Denmark. Eur Heart J Qual Care Clin Outcomes. 2020;6(1):23-31. doi:10.1093/ehjqcco/qcy063 
72. Rezaei SS, Rinner C, Ratajczak P, Grossmann W, Gall W, Wolzt M. Use of beta-blocker is associated with lower mortality in patients with coronary artery disease with or without COPD. Clin Respir J. 2018;12(12):2627-2634. doi:10.1111/crj.12968

73. Almagro P, De la Sierra A, Acosta E, et al. Spirometrically confirmed chronic obstructive pulmonary disease worsens long-term prognosis after percutaneous coronary intervention. Am J Respir Crit Care Med. 2018;197(6):824-826. doi:10.1164/rccm.201707-1389LE

74. Aboyans V, Ricco JB, Bartelink MLEL, et al. 2017 ESC Guidelines on the diagnosis and treatment of peripheral arterial diseases, in collaboration with the European Society for Vascular Surgery (ESVS). Eur Heart J. 2018;39(9):763-816. doi:10.1093/eurheartj/ ehx095

75. Kaszuba M, Śliwka A, Piliński R, et al. The comorbidity of chronic obstructive pulmonary disease and peripheral artery disease-a systematic review. COPD. 2019;16(3-4):292-302. doi:10.1080/1541 2555.2019.1653271

76. Liao KM, Kuo LT, Lu HY. Increased risk of peripheral arterial occlusive diseases in patients with chronic obstructive pulmonary disease: a nationwide study in Taiwan. Int $J$ COPD. 2019;14:14 55-1464. doi:10.2147/COPD.S202029

77. McNicholas WT, Verbraecken J, Marin JM. Sleep disorders in COPD: the forgotten dimension. Eur Respir Rev. 2013;22 (129):365-375. doi:10.1183/09059180.00003213

78. Peinado VI, Pizarro S, Barberà JA. Pulmonary vascular involvement in COPD. Chest. 2008;134(4):808-814. doi:10.1378/chest.08-0820

79. Ma Y, Tong X, Liu Y, Liu S, Xiong H, Fan H. ACE gene polymorphism is associated with COPD and COPD with pulmonary hypertension: a meta-analysis. Int J Chron Obstruct Pulmon Dis. 2018;13:2435-2446. doi:10.2147/COPD.S168772
80. Chaouat A, Naeije R, Weitzenblum E. Pulmonary hypertension in COPD. Eur Respir J. 2008;32(5):1371-1385. doi:10.1183/090319 36.00015608

81. Gredic M, Blanco I, Kovacs G, et al. Pulmonary hypertension in chronic obstructive pulmonary disease. Br J Pharmacol. 2020. doi:10.1111/bph.14979

82. Kim YR, Hwang IC, Lee YJ, Ham EB, Park DK, Kim S. Stroke risk among patients with chronic obstructive pulmonary disease: a systematic review and meta-analysis. Clinics (Sao Paulo). 2018;73. doi:10.6061/clinics/2018/e177

83. Windsor C, Herrett E, Smeeth L, Quint JK. No association between exacerbation frequency and stroke in patients with COPD. Int J COPD. 2016;11:217-225. doi:10.2147/COPD.S95775

84. Lin CS, Shih CC, Yeh CC, et al. Risk of stroke and post-stroke adverse events in patients with exacerbations of chronic obstructive pulmonary disease. PLoS One. 2017;12(1):e0169429. doi:10.1371/ journal.pone.0169429

85. Lekoubou A, Ovbiagele B. Prevalence and influence of chronic obstructive pulmonary disease on stroke outcomes in hospitalized stroke patients. eNeurologicalSci. 2017;6:21-24. doi:10.1016/j.ensci. 2016.11.007

86. Almagro P, Salvadó M, Garcia-Vidal C, et al. Recent improvement in long-term survival after a COPD hospitalisation. Thorax. 2010;65 (4):298-302. doi:10.1136/thx.2009.124818

\section{Publish your work in this journal}

The International Journal of COPD is an international, peer-reviewed journal of therapeutics and pharmacology focusing on concise rapid reporting of clinical studies and reviews in COPD. Special focus is given to the pathophysiological processes underlying the disease, intervention programs, patient focused education, and self management protocols. This journal is indexed on PubMed Central, MedLine and CAS. The manuscript management system is completely online and includes a very quick and fair peer-review system, which is all easy to use. Visit http://www.dovepress.com/testimonials.php to read real quotes from published authors. 